\title{
Thermophysical problems of laser cutting of metals
}

\author{
Anatoliy Orishich*, Victor Shulyatyev, Alexander Golyshev and Alexander Malikov \\ Khristianovich Institute of Theoretical and Applied Mechanics SB RAS, 630090, 4/1 Institutskaya \\ Str., Novosibirsk, Russia
}

\begin{abstract}
Variety and complex interaction of physical processes during laser cutting is a critical characteristic of the laser cutting of metals. Small spatial and temporal scales complicate significantly the experimental investigations of the multi-phase fluid flow in the conditions of laser cutting of metals. In these conditions, the surface formed during the cutting is an indicator determining the melt flow character. The quantitative parameter reflecting the peculiarities of the multi-phase fluid flow, is normally the roughness of the forming surface, and the minimal roughness is the criterion of the qualitative flow [1 - 2]. The purpose of this work is to perform the experimental comparative investigation of the thermophysical pattern of the multi-phase melt flow in the conditions of the laser cutting of metals with the laser wavelength of $10.6 \mu \mathrm{m}$ and $1.07 \mu \mathrm{m}$.
\end{abstract}

During the laser cutting, the local material melting and melt removal by an assistant gas jet take place. Cutting of low-carbon steel sheets is usually carried out in an oxygen jet (oxygen-assisted laser cutting). In this case, the exothermic reaction of iron oxidation is an additive energy source. The power balance for the oxygen-assisted laser cutting and the cutting with a chemically inert gas is written respectively as:

$$
\begin{array}{r}
A W+W_{o x}=W_{m}+W_{\text {cond }} \\
A W=W_{m}+W_{\text {cond }}
\end{array}
$$

where $A$ is the absorption coefficient; $W$ is the laser power; $W_{o x}$ is the power released at the exothermic reaction; $W_{m}$ is the power consumed for melting; and $W_{\text {cond }}$ is the power lost from the cut region due to thermal conductivity.

In $[2,3]$ the authors calculated the energy losses from the thermal conductivity in the laser cutting conditions $W_{\text {cond }}=\lambda_{m} t \Delta T f(\mathrm{Pe})$, where $f(\mathrm{Pe})$ is the dimensionless function of the Peclet number $\mathrm{Pe}=\left(V b \rho_{m} C_{m}\right) / \lambda_{m}, \Delta T=T^{*}-T_{0}=2000{ }^{0} \mathrm{C}$ [4], $\lambda_{m}$ is the metal thermal conductivity, $C_{m}$ is the metal heat capacity, $\rho_{m}$ is the metal density, $t$ is the steel sheet thickness, and $b$ is the cut width. Passing to dimensionless variables, we have the general expression for the energy balance: $Q+Q_{o x} \cong \operatorname{Pe}\left[1+L_{m} /\left(C_{m} \Delta T\right)\right]+f(\mathrm{Pe})$, where the following designations are used: dimensionless laser power $Q=A W /\left(\lambda_{m} t \Delta T\right)$,

${ }^{*}$ Corresponding author: laser@itam.nsc.ru 
dimensionless consumptions for metal melting $Q_{m}=W_{m} /\left(\lambda_{m} t \Delta T\right)=\operatorname{Pe}\left[1+L_{m} /\left(C_{m} \Delta T\right)\right]$, and dimensionless energy of iron oxidation $Q_{o x}=W_{o x} /\left(\lambda_{m} t \Delta T\right)$. The energy balance expression, similarly to the calculations of $[2,3]$, does not include the dependence of thermophysical parameters $\left(C_{m}\right.$ and $\left.\lambda_{m}\right)$ on the temperature; instead, their value at the average temperature $T=\left(T^{*}-T_{0}\right) / 2$ was used.

Two types of lasers were used, and their wavelengths were 10.6 and $1.07 \mu \mathrm{m}$. The ytterbium fiber laser IPG/IRE-Polus was used; its power reached $2 \mathrm{~kW}$, and the BPP parameter was $3.8 \mathrm{~mm} \cdot \mathrm{mrad}$ (BPP - Beam Parameter Product). The lens focal distance was $200 \mathrm{~mm}$. Also, the $\mathrm{CO}_{2}$ laser [5] with a self-filtering resonator was applied; its power reached $8 \mathrm{~kW}$, and the $\mathrm{BPP}=4.7 \mathrm{~mm} \cdot \mathrm{mrad}$ at the focal distance of the $\mathrm{ZnSe}$ lens of 127 and $190 \mathrm{~mm}$.

The sheets of low-carbon steel (St. 20) were cut; their thickness was 3, 5, 10, and 16 $\mathrm{mm}$, the laser power varied from 0.5 to $4 \mathrm{~kW}$, and oxygen was used as a technological gas (pressure $0.25 \mathrm{MPa})$. Under study was also cutting of stainless steel $(12 \mathrm{X} 18 \mathrm{H} 10 \mathrm{~T})$ sheets, their thickness 3,5 , and $8 \mathrm{~mm}$, nitrogen pressure $1.3-1.6 \mathrm{MPa}$.

Note that, to have experimental data for each energy flux, the optimal laser power value $W$, cutting speed $V$ and cut width $b$, were found for each thickness $t$, absorption coefficients $A$ and power losses due to the thermal conductivity were measured for these values (measurement techniques are presented in $[1,5]$. Obtained data enabled to determine the values $W_{\mathrm{m}}, A W, W_{\text {cond }}$. The value $W_{o x}$ is the power of iron oxidation exothermic reaction and was found from the expression $W_{o x}=W_{m}+W_{\text {cond }}-A W$. All optimizations involved the criterion of minimal cut roughness [1,5]. Experimental values of the energy fluxes determining the optimal laser cut are shown in Fig. 1. Evident that these values do not depend on the cut material thickness and are close to each other. It can be assumed that such an energy balance of the oxygen-assisted laser cutting of steel is a condition of the minimal surface roughness. Breach of this symmetry resulting from the non-optimal choice of cutting parameters, domination of any process, for example, excessive power of the exothermic reaction of the oxygen oxidation of iron, or jump of the power lost from the cut region due to the thermal conductivity into the surrounding metal may cause the degradation of the surface quality because of uncontrolled burning of cut region overheating.

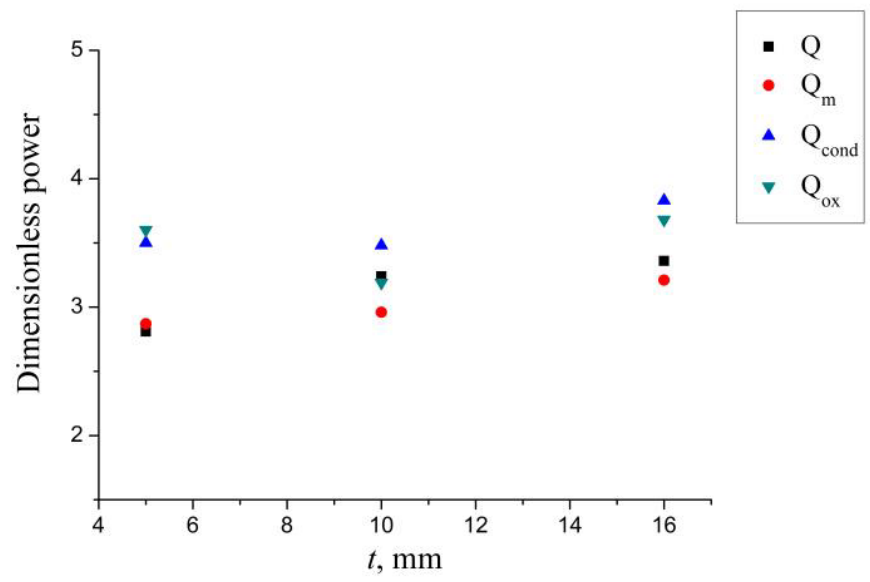

Fig. 1. Experimental values of the fluxes of dimensionless power for thicknesses 5, 10, and $16 \mathrm{~mm}$.

During the cutting with a chemically inert gas, only the utmost cutting speed guarantting the complete sheet cutting, depends on the laser power. Thus, the maximal for the fiber laser power of $2 \mathrm{~kW}$ was applied in each experiment. Opposite to the oxygenassisted laser cutting, wherein there are the power, cut speed and width optimal for each 
sheet thickness, the laser cutting with a chemically inert gas gave the qualitative cut within a wide range of speed parameters. In this case, it was necessary only to preset the optimal position of the focal spot in respect to the sheet surface at the high gas pressure (about 15 atm), for which the laser beam absorption coefficient was measured.

Figure 2 presents the generalized energy data of the cutting with an inert gas (nitrogen) as the dependence of the dimensionless parameters $Q_{a b s \_o p t}=A_{\text {opt }} W /\left(\begin{array}{lll}\lambda_{m} & t T_{m}\end{array}\right)$ on $\mathrm{Pe}_{\text {opt }}=V_{\text {opt }} b_{\text {opt }} / \gamma(\gamma-$ material thermal diffusivity). The same Figure shows the results of analysis of the energy balance of low-carbon steel cutting by the lasers with wavelengths 1.07 and $10.6 \mu \mathrm{m}$. The sum of the absorbed laser energy and energy of iron oxidation exothermic reaction was used as the absorbed energy.

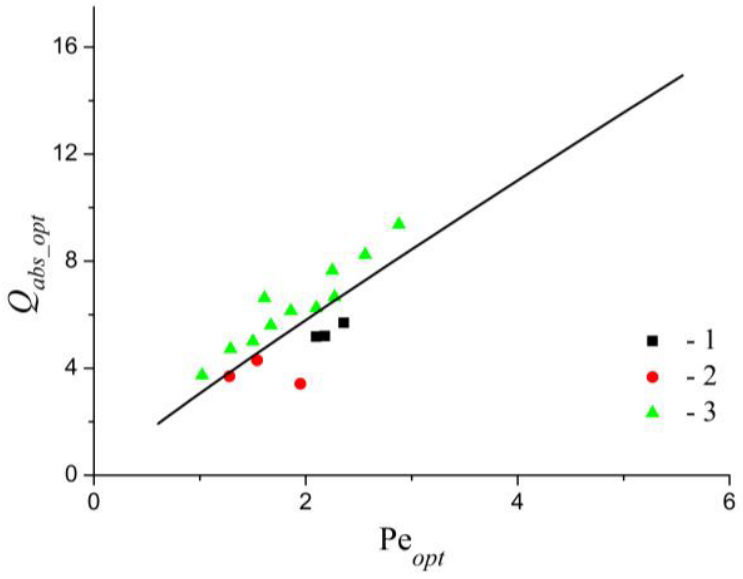

Fig. 2. Optimal value of the dimensionless power versus the optimal Peclet number during cutting of low-carbon steel by the $\mathrm{CO}_{2}$ (dots 1) and fiber (dots 2) lasers, and stainless steel by the fiber laser (dots 3 ). The line is plotted by the formula (3).

Evident that utilization of the dimensionless parameters permit generalizing all variety of the experimental data regardless the metal thickness, laser power and type, into one common dependence approaching to $Q_{a b s \_o p t} \sim \mathrm{Pe}_{\text {opt }}$. The inclination of the experimental dependence in Fig. 2 enables to evaluate the average energy contribution in the volume unit of the metal removed from the cut region: $E_{a b s \_p t} \approx 26 \mathrm{~J} / \mathrm{mm}^{3}$. Note that, during the cutting with the inert gas, all this energy should be provided by the laser radiation, whereas in the presence of oxygen, $50 \%$ of this contribution is provided by the laser beam $\left(13 \mathrm{~J} / \mathrm{mm}^{3}\right)$ and $50 \%$ - by the exothermic reaction of iron oxidation.

Let us consider the applicability of the theoretical energy balance to describe the experimental data. According to [3], the expression $W_{\text {cond }}^{*}=f(\mathrm{Pe})=W_{\text {cond }}\left(\lambda_{m} t \Delta T\right)=3.2(\mathrm{Pe} / 2)^{0.868}$ can be used for the evaluation of thermal losses. In [2], another dependence of the thermal losses on the Peclet number was found: $W_{\text {cond }}^{*}=f(\mathrm{Pe})=W_{\text {cond }} /\left(\lambda_{m} t \Delta T\right)=3.2(\mathrm{Pe} / 2)^{0.3}$. Evident that only the model proposed in [3] can satisfy the experimental dependence $Q_{a b s \_o p t} \sim \mathrm{Pe}_{\text {opt }}$. Substituting theoretical thermal losses in the energy balance, we have:

$$
Q_{a b s \_x}+Q_{a b s \_ \text {inert }}=1.3 \mathrm{Pe}+3.2(\mathrm{Pe} / 2)^{0.868}
$$

where $Q_{a b s o x}=Q+Q_{o x} \cong 2 Q$.

Opposite to [3], the Peclet number in the resulting expressions was calculated with the channel diameter. Material properties are regarded by the parameter $L_{m} /\left(C_{m} \Delta T\right)$, which equaled to 0.284 and 0.316 for the low-carbon and stainless steels, respectively. During the calculation of the power balance (5), the average value 0.3 was used. Figure 2 shows with 
the solid line the results of calculation of the power balance. Thus, comparison with the experiment demonstrates that the model of the thermal losses proposed in [3] describes the experimental data more adequately.

\section{References}

1. A.A. Golyshev, A.G. Malikov, A.M. Orishich, V.B. Shulyatyev, Quantum Electronics 45, 873 (2015)

2. W. Schulz, D. Becker, J. Franke, R. Kemmerling, G. Herziger, J. Phys. D: Appl. Phys. 26, 1357 (1993)

3. J.M. Prusa, G. Venkitachalam, P.A. Molian, Int. J. Machine Tools and Manufacture 39, 537 (1999)

4. J. Powell, D. Petring, R.V. Kumar, S.O. Al-Mashikhi, A.F.H. Kaplan, K.T. Voisey J. Phys. D: Appl. Phys. 42, 015504 (2009)

5. V.M. Fomin, A.A. Golyshev, A.G. Malikov, A.M. Orishich, V.B. Shulyatyev, J. Appl. Mech. Techn. Phys. 56, 726 (2015) 